\title{
SOME NEW RESULTS ON THE CHU DUALITY OF DISCRETE GROUPS
}

\author{
SALVADOR HERNÁNDEZ AND TA-SUN WU
}

\begin{abstract}
This paper deals mainly with the Chu duality of discrete groups. Among other results, we give sufficient conditions for an $F C$ group to satisfy Chu duality and characterize when the Chu quasi-dual and the Takahashi quasi-dual of a group $G$ coincide. As a consequence, it follows that when $G$ is a weak sum of a family of finite simple groups, if the exponent of the groups in the family is bounded then $G$ satisfies Chu duality; on the other hand, if the exponent of the groups goes to infinite then the Chu quasi-dual of $G$ coincide with its Takahashi quasi-dual. We also present examples of discrete groups whose Chu quasi-duals are not locally compact and examples of discrete Chu reflexive groups which contain non-trivial sequences converging in the Bohr topology of the groups. Our results systematize some previous work and answer some open questions in the subject [2, 16] 3].
\end{abstract}

\section{INTRODUCTION}

It is a consequence of the celebrated Gel'fand and Raǐkov Theorem that the set of all unitary representations of a locally compact group $G$ contains the information necessary to recover the topological and algebraic structure of the group (see [5]). However, in general, such representations may not be finite dimensional. The result is that if we form a dual space associated to the set of all representations, we obtain an object whose structure is very involved. In [2] Chu, motivated by the work of Hochschild and Mostow [10] for compact groups, considered the groups with enough finite dimensional representations to separate the points (the so-called maximally almost periodic groups) and established a duality theory within this class that extends both Pontryagin and Tannaka-Krein dualities for locally compact Abelian groups and compact groups respectively. Chu defined the space $G^{x}$ consisting of all finite dimensional representations on $G$ equipped with the compact open topology. Then he used the dual structure of $G^{x}$ inherited from $G$ to form a bidual object $G^{x x}$ consisting of certain continuous mappings on $G^{x}$ that behave nicely with respect to the algebraic structure of $G^{x}$. It turns out that $G^{x x}$ is a topological group with nice features, which is called the Chu quasi-dual group of $G$. When $G$ is topologically isomorphic to $G^{x x}$ it is said that $G$ satisfies Chu (unitary) duality or $G$ is Chu reflexive (is Chu for short). Thus, investigating the Chu duality of locally compact groups is equivalent to identifying the locally compact groups that can be recovered from their set of finite

1991 Mathematics Subject Classification. Primary 22D35, 43A40; Secondary 22D05, 22D10, $54 \mathrm{H} 11$.

Key words and phrases. locally compact group, discrete group, Chu duality, unitary duality, Bohr compactification.

The first named author acknowledges partial financial support by the Spanish Ministry of Science (including FEDER funds), grant MTM2004-07665-C02-01; and the Generalitat Valenciana, grant GV04B-019 . 
dimensional unitary representations. Chu set the basis for this duality theory but, after Chu's seminal paper, there have been several important contributions devoted to developing this theory. We shall mention some of them below. Nevertheless, many important questions along this line of research are still open. Some of them were asked by Chu himself. We mention just a representative one here. Does the discrete free group with two generators satisfy Chu duality?

This paper deals mainly with the Chu duality of discrete groups. Among other results, we give sufficient conditions for an $F C$ group to satisfy Chu duality and characterize when the Chu quasi-dual and the Takahashi quasi-dual of a group $G$ coincide. As a consequence, it follows that when $G$ is a weak sum of a family of finite simple groups, if the exponent of the groups in the family is bounded then $G$ satisfies Chu duality; on the other hand, if the exponent of the groups goes to infinite then the Chu quasi-dual of $G$ coincide with its Takahashi quasi-dual. We also present examples

of discrete groups whose Chu quasi-duals are not locally compact and examples of discrete Chu reflexive groups which contain non-trivial sequences converging in the Bohr topology of the groups. Our results systematize some previous work and answer some open questions in the subject (see [2, 16, 3]).

\section{Basic Definitions And Facts}

In principle, all groups are assumed to be locally compact Hausdorff and maximally almost periodic (MAP). That is, locally compact groups that can be continuously injected into compact groups. For any group $G$, the symbol $G^{\prime}$ means its commutator subgroup. The group $G$ has finite exponent if the orders of the elements of $G$ are finite and bounded. The exponent of $G$, denoted $\exp (G)$ is the least common multiple of all the orders of elements in $G$.

Let $G$ be a topological group, denote by $G_{n}^{x}$ the set of all continuous $n$-dimensional unitary representations of $G$, i. e., the set of all continuous homomorphisms of $G$ into the unitary group $U(n)$, equipped with the compact-open topology. It follows from a result due to Goto [7] that $G_{n}^{x}$ is a locally compact and uniformizable space. The space $G^{x}=\sqcup_{n<\omega} G_{n}^{x}$ (as a topological sum) is called the Chu dual of $G$ [2].

We now recall the basic notions of the Chu (or unitary) duality. Its main feature is the construction of a bidual of $G$ from the Chu dual $G^{x}$. This bidual consists of the so-called quasi-representations. If we define $\mathcal{U}=\sqcup_{n<\omega} \mathcal{U}(n)$ (topological sum), a quasi-representation of $G^{x}$ is a continuous mapping $p: G^{x} \longrightarrow \mathcal{U}$ conserving the main operations between unitary representations: direct sums, tensor products, unitary equivalence and sending the elements of $G_{n}^{x}$ into $U(n)$ for all $n \in \mathbb{N}$ (see [2] or [8] for details). The set of all quasi-representations of $G$ equipped with the compact-open topology is a topological group with pointwise multiplication as the composition law, called the Chu quasi-dual group of $G$ and denoted by $G^{x x}$. Thus, a neighborhood base of the identity in $G^{x x}$ consists of sets of the form $\left[K_{n}, V\right]=\left\{p \in G^{x x}: p\left(K_{n}\right) \subset V\right\}$, where $V$ is any neighborhood of the identity in $U(n)$ and $K_{n}$ is any compact subset of $G_{n}^{x}, n \in \mathbb{N}$. It is easily verified that the evaluation map $\epsilon_{G}: G \longrightarrow G^{x x}$ is a group homomorphism which is a monomorphism if and only if $G$ is MAP. The group $G$ satisfies Chu duality when the evaluation map $\epsilon_{G}$ is an isomorphism of topological groups. If the evaluation map is only an algebraic isomorphism, we say that $G$ is Chu semi-reflexive. In this terminology it was shown in [2], that LCA groups and 
compact groups satisfy Chu duality (indeed Chu duality reduces to Pontryagin duality and to Tannaka duality respectively for such groups). Here, the group $\epsilon_{G}(G)$ is always assumed to be equipped with the topology inherited from $G^{x x}$.

Two $n$-dimensional representations $D_{1}$ and $D_{2}$ of $G$ are said (unitarily) equivalent to each other when there is $U$ in $U(n)$ such that $D_{1}(x)=U^{-1} D_{2}(x) U$ for all $x \in G$. This notion sets an equivalence relation on $G_{n}^{x}$ that we denote with the symbol " $\sim$ ".

Let $\operatorname{Irr}_{n}(G)$ denote the set of all irreducible representations of dimension $n$. In addition to the unitary dual, it will be useful to consider the set $\widehat{G}_{n}=\operatorname{Irr}_{n}(G) / \sim$, which is the quotient space formed by the equivalence classes of irreducible representations of dimension $n$.

The Bohr compactification of an arbitrary topological group can be defined as a pair $(b G, b)$ where $b G$ is a compact Hausdorff group and $b$ is a continuous homomorphism from $G$ onto a dense subgroup of $b G$ with the following universal property: for every continuous homomorphism $h$ from $G$ into a compact group $K$ there is a continuous homomorphism $h^{b}$ from $b G$ into $K$ extending $h$ in the sense that $h=h^{b} \circ b$, that is, making the following diagram commutative:

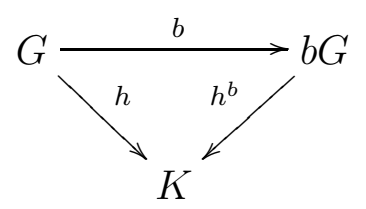

The group $b G$ is essentially unique and is also referred to as the Bohr compactification of $G$. Heyer [8, V, $\S 14]$ contains a careful examination of $b G$ and its properties. The group $G$ may be equipped with the topology induced by the above homomorphism $b$, the so-called Bohr topology. The Bohr topology is Hausdorff precisely when $G$ is maximally almost periodic ( $M A P$ group), equivalently, when $b$ is one-to-one. Here, we will be mainly concerned with this class of groups; these turn out to be the groups whose finite dimensional representations separate points. The Bohr topology of a group $G$ may also be defined as the one that $G$ inherits from $\mathcal{U}^{G^{x}}$ (the topology of pointwise convergence on $G^{x}$ ). The symbol $G^{b}$ stands for $G$ equipped with the Bohr topology. Clearly, each member of $G^{x}$ defines a continuous mapping on $G^{b}$ that extends to $b G$. Thus, the representation spaces $G^{x}$ and $(b G)^{x}$ have exactly the same underlying set. As a consequence, the Chu quasi-dual group $G^{x x}$ is always algebraically embedded into $b G$.

We now collect some well-known facts about Chu duality theory (see [, 9, 17, 19])

Proposition 2.1. The following assertions hold for any locally compact MAP group G.

(1) The space $G_{n}^{x}$ is locally compact for each $n \in \mathbb{N}$, so is $G^{x}$. If $G$ is discrete (resp. metrizable), then $G_{n}^{x}$ is compact (resp. hemicompact) and therefore $G^{x}$ is $\sigma$-compact.

(2) If $G$ is compact then the quotient space $G^{x} / \sim$ is discrete.

(3) If $G$ satisfies the second axiom of countability then $G_{n}^{x}$ satisfies the second axiom of countability. So $G^{x}$ is metrizable.

(4) The group $G$ is maximally almost periodic if and only if $\epsilon_{G}$ is injective. 
(5) $G^{x x}$ is complete with respect to uniformity of uniform convergence on compact subsets.

(6) If $G$ is second countable then $G^{x x}$ is a second countable complete metric space. As a consequence, if the evaluation map $\epsilon_{G}$ is onto then $G$ is Chu.

In some cases, the Chu quasi-dual of a group $G$ coincides with its Bohr compactification $b G$. Here on, we denote by $C(K, U(n))$ the space of all continuous functions from a topological space $K$ into $U(n)$.

Proposition 2.2. Let $G$ be a locally compact group. It holds that $G^{x x}$ is topologically isomorphic to $b G$ if and only if $\widehat{G}_{n}$ is discrete for any $n \in \mathbb{N}$.

Proof. Sufficiency: This is Theorem 4.4 of [6].

Necessity: Let $\alpha: G_{n}^{x} \longrightarrow\left(G^{x x}\right)_{n}^{x}$ the canonical evaluation map defined by $\alpha(D)(p)=$ $p(D)$ for all $D \in G_{n}^{x}$ and $p \in G^{x x}$. We first check that $\alpha$ is continuous and injective. For the continuity, let $\left\{D_{j}\right\}$ be a net converging to $D$ in $G_{n}^{x}$. If $K$ is a compact subset of $G^{x x}$, since $G_{n}^{x}$ is locally compact, it follows that $K$ is an equicontinuous subset of $C\left(G_{n}^{x}, U(n)\right)$. Hence, the net $\left\{\alpha\left(D_{j}\right)\right\}$ converges to $\alpha(D)$ uniformly on $K$. This yields the continuity of $\alpha$. For the injectivity, suppose that $D, E$ belong to $G_{n}^{x}$ and $\alpha(D)=\alpha(E)$. Then $p(D)=p(E)$ for all $p \in G^{x x}$. In particular, $D(g)=E(g)$ for all $g \in G$. Thus, $\mathrm{D}=\mathrm{E}$.

Now, we define $\bar{\alpha}: G_{n}^{x} / \sim \longrightarrow\left(G^{x x}\right)_{n}^{x} / \sim$ so that the following diagram commutes

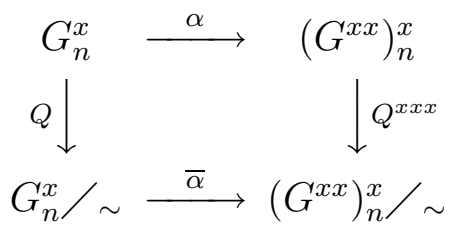

where $Q$ and $Q^{x x x}$ are the canonical quotient mappings. In order to check that $\bar{\alpha}$ is properly defined, suppose that $E$ and $D$ are in $G_{n}^{x}$ and $E \sim D$. Then, there is $A \in U(n)$ such that $D(g)=A E(g) A^{-1}$ for all $g \in G$. Since, by hypothesis, $G^{x x}$ is topologically isomorphic to $b G$, it follows that $G$ is dense in $G^{x x}$ with respect to the topology of pointwise convergence on $G^{x}$. Thus, $D(p)=A E(p) A^{-1}$ for all $p \in G^{x x}$. Therefore, $\alpha(E) \sim \alpha(D)$ and this implies that $\bar{\alpha}$ is well defined. The fact that $Q$ and $Q^{x x x}$ are quotient mappings yields the continuity of $\bar{\alpha}$. Finally, let us see that $\bar{\alpha}$ is also injective. Suppose $E$ and $D$ in $G_{n}^{x}$ such that $\bar{\alpha}(Q(E))=\bar{\alpha}(Q(D))$. Then there is $A \in U(n)$ such that $\alpha(D)(p)=A \alpha(E)(p) A^{-1}$ for all $p \in G^{x x}$. Thus, $D(g)=A E(g) A^{-1}$ for all $g \in G$ and, as a consequence, $E \sim D$. This gives the injectivity of $\bar{\alpha}$. On the other hand, since $G^{x x}$ is compact, it follows from Proposition 2.1 (2) that $\left(G^{x x}\right)_{n}^{x} / \sim$ is discrete. Since $\bar{\alpha}$ is continuous and injective, it follows that $G_{n}^{x} / \sim$ is also discrete. This completes the proof because $\widehat{G}_{n}$ is a subspace of the latter.

We notice that in general the surjectivity of $\epsilon_{G}: G \longrightarrow G^{x x}$ does not imply that $\epsilon_{G}$ is a homeomorphism. As an example (due to Moran) let $\left(p_{n}\right)$ be an infinite sequence of distinct primes numbers $\left(p_{n}>2\right)$, and let $G_{n}$ be the projective special linear group of dimension two over the Galois field $\left(G F\left(p_{n}\right)\right)$ of order $p_{n}$. Let $G=\prod_{n=1}^{\infty} G_{n}$ with discrete topology. Then $G^{x x}=\prod_{n=1}^{\infty} G_{n}$ is compact (with product topology), (cf. [12]). 


\section{FC Groups}

Let $G$ be a (discrete) group. We say that $G$ is an $F C$ group if every conjugacy class of $G$ is finite, i.e., for all $g \in G$, we have that $\mathcal{O}_{x}:=\left\{h g h^{-1}: h \in G\right\}$ is finite. We say that $g \in G$ is central in $G$ if $g h=h g$ for all $h \in G$. The set of all central elements of $G$ is a normal subgroup called the center, $Z(G)$, of $G$. A subgroup $H$ of $G$ is called central in $G$ if $H \subset Z(G)$. In the sequel, the symbol mdus $(G)$ denotes the minimal natural number $n$ such that the unitary representations of dimension less or equal than $n$ separate the points in $G$. Our main goal in this section is to prove the following result.

Theorem 3.1. Let $G$ be an $F C$ torsion group and suppose there is $N \in \mathbb{N}$ such that $\exp \left(G^{\prime}\right) \leq N$ and $\operatorname{mdus}(G / H) \leq N$ for all normal subgroup $H$ of $G$ that is co-finite in $G^{\prime}$. Then the group $G$ is Chu reflexive.

As a corollary, it follows the following result about direct sums which are not necessarily torsion.

Corollary 3.2. Let $G=\sum_{i \in I} F_{i}$ equipped with the discrete topology such that $F_{i}^{\prime}$ is finite for all $i \in I$. Suppose further that there is $N \in \mathbb{N}$ with $\operatorname{mdus}\left(F_{i}\right) \leq N$ and $\exp \left(F_{i}{ }^{\prime}\right) \leq N$, for all $i \in I$. Then the group $G$ is Chu reflexive.

We notice that the constraints on $\operatorname{mdus}(G)$ and $\exp \left(G^{\prime}\right)$ are only needed in Proposition 3.11 below. The proof of Theorem 3.1 is split in several partial results. Firstly, we need some definitions.

Let $G$ be a topological group and let $H$ be a subgroup of it. We define the $C h u$ quasi-dual of $H$ in $G$ to be the group $G^{x x} \cap c l_{b G} H$ We denote this group by $(H, G)^{x x}$.

We say that $H$ is $C h u$ semi-reflexive in $G$ when $H$ coincides algebraically with $(H, G)^{x x}$.

Proposition 3.3. Let $G$ be a topological group and let $H$ be normal subgroup which is Chu semi-reflexive in $G$. If $G / H$ is Chu semi-reflexive then $G$ is Chu semi-reflexive.

Proof. Consider the exact sequence

$$
1 \longrightarrow H \stackrel{i}{\longrightarrow} G \stackrel{j}{\longrightarrow} G / H \longrightarrow 1
$$

and the dual maps (in the category of pointed spaces)

$$
1 \longrightarrow(G / H)^{x} \stackrel{j^{x}}{\longrightarrow} G^{x} \stackrel{i^{x}}{\longrightarrow} G_{\mid i(H)}^{x} \longrightarrow 1
$$

where $i^{x}$ and $j^{x}$ are both continuous, the map $i^{x}$ is onto, and the inverse image under $i^{x}$ of the set of representations of $G$ which are trivial on $H$, is $(G / H)^{x}$.

Repeating the process above, one can also obtain the Chu quasi-dual sequence.

$$
1 \longrightarrow(H, G)^{x x} \stackrel{i^{x x}}{\longrightarrow} G^{x x} \stackrel{j^{x x}}{\longrightarrow}(G / H)^{x x} \longrightarrow 1
$$

Next we verify that this sequence is exact.

Firstly, observe that, since $\epsilon$ is a natural transformation, the following diagram is commutative 


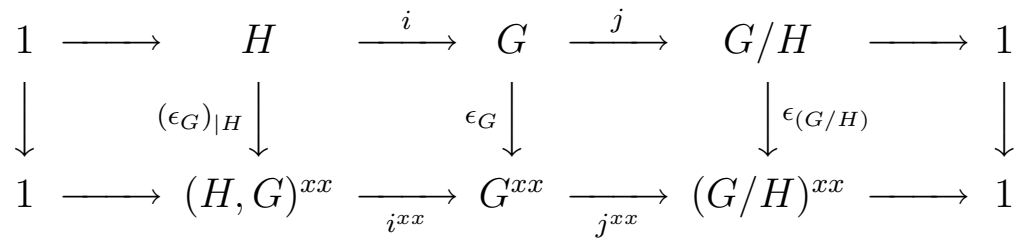

where $\left(\epsilon_{G}\right)_{\mid H}$ and $\epsilon_{(G / H)}$ are known to be onto algebraic isomorphisms and $\epsilon_{G}$ is 1-to-1.

Being and embedding, the map $i^{x x}$ is 1-to-1 and since $\epsilon_{(G / H)}$ is bijective and the diagram above is commutative, it follows that that $j^{x x}$ is onto.

Next we show that $\operatorname{Im}\left(i^{x x}\right)=\operatorname{ker} j^{x x}$. Indeed, $i^{x x}(H, G)^{x x}=\left(\epsilon_{G} \circ i \circ \epsilon_{H}^{-1}\right)(H, G)^{x x}=$ $\epsilon_{G}(i(H)) \subset \epsilon_{G}(\operatorname{ker} j) \subset \operatorname{ker} j^{x x}$. On the other hand, let $p$ be an arbitrary element of $k e r j^{x x}$. We have that $j^{x x}(p)(E)=p\left(j^{x}(E)\right)=p(E \circ j)=I_{n}$ for all $E \in(G / H)_{n}^{x}$ and for all $n<\omega$. We must check that $p$ is in $c l_{b G} H=K$. Suppose the contrary and let $\pi: b G \longrightarrow b G / K$ the canonical quotient mapping. We have that $\pi(p) \neq 1_{b G / K}$. Hence, there is $E \in(b G / K)_{n}^{x}$ such that $E(\pi(p)) \neq I_{n}$. Now, it is readily seen that $b(G / H)$ is topologically isomorphic to $b G / K$. Thus, $E_{\mid(G / H)}$ belongs to $(G / H)_{n}^{x}$ and we have that $p\left(j^{x}\left(E_{\mid(G / H)}\right)\right)=p\left(E_{\mid(G / H)} \circ j\right)=p(E \circ \pi)=E(\pi(p)) \neq I_{n}$. This is a contradiction which completes the proof of the exactness of the quasi-dual sequence.

Applying the well-known five-lemma to the diagram above, using that $\epsilon_{H}$ and $\epsilon_{(G / H)}$ are isomorphisms onto, we obtain that $\epsilon_{G}$ is an algebraic onto isomorphism.

Corollary 3.4. Let $G$ be a $L C$ topological group. If $G$ ' is Chu semi-reflexive in $G$ then the group $G$ is Chu semi-reflexive.

Next lemma is due to $\mathrm{Wu}$ and Riggins (see [21, p. 462]) but we include a short proof of it for the reader's sake. We recall that when $G$ is an FC group we have that $\mathcal{O}_{x}$ is finite for all $x \in G$. Thus, $F_{x}$, the isotropy group of $x$, is co-finite in $G$.

Lemma 3.5. Let $G$ be an $F C$ group. Given an arbitrary element $D$ of $G_{n}^{x}, n \in \mathbb{N}$, we have $Z(D(G))$ is co-finite in $D(G)$.

Proof. Let $K$ be the closure of $D(G)$ in $\mathrm{U}(\mathrm{n})$. Then $K$ is a compact Lie group with a dense $F C$ subgroup. Thus, $\mathcal{O}_{x}$ is finite for all $x \in D(G)$. In particular, if $K_{0}$ denotes the connected component of $K$, we have $\mathcal{O}_{x} \cap K_{0}=\{x\}$ for all $x \in D(G) \cap K_{0}$. Since $D(G)$ is dense in $K$, this means that $K_{0} \subset Z(K)$. From the compactness of $K$, it follows that $K_{0}$ is co-finite in $K$ and, as a consequence, that $Z(D(G))$ is co-finite in $D(G)$.

Lemma 3.6. Let $G$ be an FC group. Given an arbitrary element $D$ of $G_{n}^{x}, n \in \mathbb{N}$, there is a co-finite normal subgroup $N$ of $G$ such that $D\left(N^{\prime}\right)=\left\{I_{n}\right\}$. Moreover, the group $G^{\prime} / N^{\prime}$ is finite.

Proof. Since $G$ is an FC group, by the lemma above, we have that $L=Z(D(G))$ is cofinite in $D(G)$. Thus, $D(G) / L=\left\{D\left(x_{1}\right) L, \ldots, D\left(x_{m}\right) L\right\}$. For each $x_{i}, 1 \leq i \leq m$, let $F_{i}$ be the isotropy group of $x_{i}$. The subgroup $F=\cap_{i=1}^{m} F_{i}$ is co-finite and $a x_{i} a^{-1}=x_{i}$ for $a \in F, 1 \leq i \leq m$. Hence $D(F) \subset Z(D(G))$, what yields $D\left(F^{\prime}\right)=\left\{I_{n}\right\}$. Now, if we take $A$ to be the normal subgroup generated by $\left\{x_{1}, \ldots x_{m}\right\}$, then its centralizer in $G$, the subgroup $C_{G}(A)$, is a co-finite normal subgroup of $G$ with $F \supset C_{G}(A)$ (see [21. p. 462]). Thus, it suffices to take $N=C_{G}(A)$ and the first part of proof is done. Let us verify now that $G^{\prime} / N^{\prime}$ is finite. Indeed, since $N$ is co-finite in $G$, we have 
$\frac{G}{N}=\left\{x_{1} N, \ldots, x_{l} N\right\}$, with $x_{i} \in G, 1 \leq i \leq l$. On the other hand, $G^{\prime}$ is generated by elements of the form $\left[x_{i} a, x_{j} b\right]$, with $a, b \in N$. Now, because $N$ is normal in $G$, we know that $N^{\prime}$ is also normal in $G$. Hence, we have

$$
\begin{aligned}
& {\left[x_{i} a, x_{j} b\right] N^{\prime}=} \\
& \left(x_{i} a x_{j} b a^{-1} x_{1}^{-1} b^{-1} x_{j}^{-1}\right) N^{\prime}= \\
& \left(x_{i}\left(a x_{j} a^{-1}\right)[a, b]\left(b x_{i}^{-1} b^{-1}\right) x_{j}^{-1}\right) N^{\prime}= \\
& \left(x_{i}\left(a x_{j} a^{-1}\right)\left(b x_{i}^{-1} b^{-1}\right) x_{j}^{-1}\right) N^{\prime}
\end{aligned}
$$

Since $G$ is an FC group, we obtain that there are only finitely many elements of the form $\left[x_{i} a, x_{j} b\right] N^{\prime}$, with $a, b \in G$ and $x_{i}, x_{j} \in\left\{x_{1}, \ldots, x_{l}\right\}$, which proves that $\frac{G^{\prime}}{N^{\prime}}$ is finitely generated. Now, by [18, 14.5.9], $G^{\prime}$ is torsion and, as a consequence, so is $\frac{G^{\prime}}{N^{\prime}}$. Hence, the quotient group $\frac{G^{\prime}}{N^{\prime}}$ is finitely generated, torsion and FC. Applying [18, 14.5.7], we obtain that $\frac{G^{\prime}}{N^{\prime}}$ is finite, which completes the proof.

Using that $N^{\prime} \subset \operatorname{Ker} D$ in the lemma above, it follows.

Corollary 3.7. Let $G$ be an $F C$ group. Then for any element $D$ of $G_{n}^{x}, n \in \mathbb{N}$, we have that $G^{\prime} /\left((\operatorname{Ker} D) \cap G^{\prime}\right)$ is finite.

The proof of Lemma 3.6 may be adapted easily to obtain also following result.

Lemma 3.8. Let $G=\sum_{i \in I} F_{i}$, where $F_{i}^{\prime}$ is finite for all $i \in I$. Then, for each $D \in \operatorname{Hom}(G, U(n))$, there is a finite subset $J \subseteq I$ such that, if $x$ and $y$ belong to $G^{\prime}$ and $x_{\mid J}=y_{\mid J}$, then we have that $D(x)=D(y)$.

Let $G$ be an $F C$ group and consider a family $\mathcal{B}$ of normal subgroups of $G$ satisfying: (1) for every $A \in \mathcal{B}$ we have that $G^{\prime} /\left(A \cap G^{\prime}\right)$ is finite; (2) for all finite subset $F$ of $G$, and $D \in G_{n}^{x}$ with $D(F)=\left\{I_{n}\right\}$, there is $A \in \mathcal{B}$ such that $F \subset A$ and $A \subset K e r D$; and (3) the set $\mathcal{B}$ is directed under inverse inclusion. For instance, we have just verified that, if $\mathcal{D} \subset G^{x}$ is such that $\mathcal{D}$ contains $G_{1}^{x}$ and separates the points of any quotient $G / L$, with $L$ a normal subgroup of $G$ such that $L \cap G^{\prime}$ is co-finite in $G^{\prime}$, then one can take $\mathcal{B}$ to be the family $\left\{\cap_{i=1}^{n} \operatorname{Ker} D_{i}: D_{i} \in \mathcal{D}, n \in\right.$ $\mathbb{N}\}$. Now, consider the projective system $\left\{\left(G / A, f_{A B}\right): A, B \in \mathcal{B}\right\}$, where $f_{A B}$ : $G / B \longrightarrow G / A$ is the canonical homomorphism naturally defined when $B \subset A$ and equip each group $G / A$ with the weak topology generated by $(G / A)^{x}$. Define $H$ to be

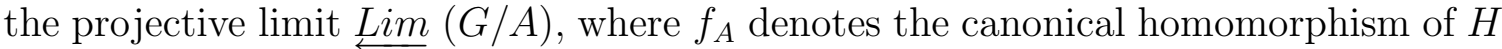
onto $G / A$ and observe that, since its topology is defined by finite dimensional unitary representations, the group $H$ is totally bounded. On the other hand, according to the definition of $\mathcal{B}$, the subgroup of $H$, defined as $K=\underline{\operatorname{Lim}}\left(G^{\prime} /\left(A \cap G^{\prime}\right)\right)$ is profinite and, therefore, compact.

Proposition 3.9. Let $G$ a maximally almost periodic $F C$ group and let $\mathcal{B}$ be a family of subgroups of $G$ satisfying the assertions (1)- (3), as defined above. Then $\mathrm{cl}_{b G} G^{\prime}$ is topologically isomorphic to $K=\underline{\operatorname{Lim}}\left(G^{\prime} /\left(A \cap G^{\prime}\right)\right)$.

Proof. Let $\pi_{A}: G \longrightarrow G / A$ the canonical quotient and consider the map $\psi: G \rightarrow H=$ $\operatorname{Lim}(G / A)$, which is defined by $f_{A} \circ \psi=\pi_{A}$. Then the map $\psi$ is a homomorphism trivially continuous when we consider the Bohr topology on $G$. By the universal property of the Bohr compactification, $\psi$ can be extended to a continuous group homomorphism $\bar{\psi}: b G \rightarrow \bar{H}$, with $\bar{H}$ being the Weil completion of the group $H$. 
Since $\psi\left(G^{\prime}\right)$ is dense in the compact subgroup $K=\underbrace{}_{\operatorname{Lim}}\left(G^{\prime} /\left(A \cap G^{\prime}\right)\right)$ of $H$, it follows that $\bar{\psi}$ is a quotient homomorphism from $c l_{\underline{b} G} G^{\prime}$ onto $K$. In order to prove that $\bar{\psi}$ is an isomorphism, it suffices to show that $\bar{\psi}$ is injective on $c_{b G} G^{\prime}$. Now, if $g \in c l_{b G} G^{\prime}$ and $g \neq 1$, then there exists $n \in \mathbb{N}$ and $D \in(b G)_{n}^{x}=G_{n}^{x}$ such $D(g) \neq I_{n}$. By Corollary [3.7. we know that the group $G^{\prime} /\left((\operatorname{Ker} D) \cap G^{\prime}\right)$ is finite. By (2), we can take $A \in \mathcal{B}$ such that $A \subset G \cap \operatorname{Ker} D$. Clearly, the representation $D$ factorizes through $E: b G /\left(c l_{b G} A\right) \longrightarrow U(n)$. Thus, if $\bar{f}_{A}$ denotes the canonical extension of $f_{A}$, the following diagram

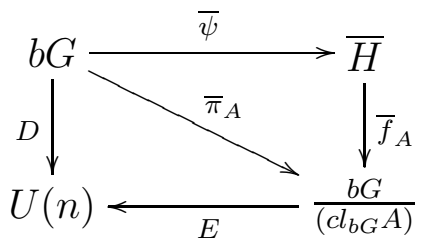

commutes.

This yields $\bar{f}_{A}(\bar{\psi}(g)) \neq 1$ and shows the injectivity of $\bar{\psi}$, which completes the proof.

As a consequence of Proposition 3.9, we obtain.

Corollary 3.10. Let $G=\sum_{i \in I} F_{i}$, where $F_{i}^{\prime}$ is finite for all $i \in I$. Then we have that $\mathrm{cl}_{b G} G^{\prime}$ is topologically isomorphic to $K=\prod_{i \in I} F_{i}^{\prime}$. As a consequence, it follows that $G^{\prime}$ is Bohr closed in $G$.

Proof. It is enough to take $\mathcal{B}=\left\{\sum_{i \in I \backslash J} F_{i}: J \subset I\right.$, and $J$ finite $\}$

Proposition 3.11. Let $G$ be an $F C$ torsion group and suppose there exist a family $\mathcal{B}$ of normal subgroups of $G$ satisfying (1)-(3), as defined above. Assume further that there is $N \in \mathbb{N}$ such that $\exp \left(G^{\prime}\right) \leq N$ and mdus $(G / B) \leq N$ for all $B \in \mathcal{B}$. Then $G^{\prime}$ is Chu semi-reflexive in $G$.

Proof. It suffices to verify that $G^{\prime}$ is Chu semi-reflexive in $G$. Let $g=\left(g_{A}\right)$ be an element of $c l_{b G} G^{\prime} \cap G^{x x} \cong \underline{\operatorname{Lim}}\left(G^{\prime} /\left(A \cap G^{\prime}\right)\right) \cap G^{x x}$ and suppose that $g \notin G^{\prime}$. Firstly, observe that $g \notin G$ since $G^{\prime}$ is Bohr closed in $G$ (indeed, if $g \in G \backslash G^{\prime}$, then there is a character $\chi$ on $G$ such that $\chi(g) \neq 1)$. Now, for any arbitrary finite subset $F$ of $G$, since $G$ is an $F C$ torsion group, we know that the normal closure $F^{G}$ of $F$ in $G$ is finite. Thus, the same happens with its normal closure in $b G$. That is, we have $F^{b G}=F^{G}$. Now, the compact group $b G / F^{G}$ is MAP and, since $g \notin F^{G}$, we can find $D \in(b G)_{n}^{x}$ such that $D\left(F^{G}\right)=\left\{I_{n}\right\}$ and $D(g) \neq I_{n}$. By (2), take $B_{F} \in \mathcal{B}$ such that $F^{G} \subset B_{F}$ and $B_{F} \subset(G \cap K e r D)$. It follows that $f_{B_{F}}\left(\psi\left(F^{G}\right)\right)=1$ but $\bar{f}_{B_{F}}(\bar{\psi}(g)) \neq 1$. Therefore, by hypothesis, there is $\tilde{E}_{F} \in\left(G / B_{F}\right)_{N}^{x}$ such that $\tilde{E}_{F}\left(\bar{f}_{B_{F}}(\bar{\psi}(g)) \neq I_{N}\right.$. Let $V$ be a neighborhood of the identity in $U(N)$ that contains no non-trivial elements of order $\leq N$. Since $\exp \left(G^{\prime} / B_{F}\right) \leq N$ and $\bar{\psi}_{B_{F}}(g) \in G^{\prime} / B_{F}$, we have $\tilde{E}_{F}\left(\bar{\psi}_{B_{F}}(g) \notin V\right.$. Set $E_{F}=\tilde{E}_{F} \circ \psi_{B_{F}}$ for all finite subset $F$ of $G$ and let $\mathcal{F}$ be the set consisting of all finite subsets of $G$, ordered under inclusion. Since $E_{F}\left(F^{G}\right)=\left\{I_{N}\right\}$ for all $F \in \mathcal{F}$, it is easily verified that the net $\left\{E_{F}: F \in \mathcal{F}\right\}$ converges to the identity representation in $G_{N}^{x}$. On the other hand, since $g \in(\underbrace{\operatorname{Lim}}_{\operatorname{Lim}}\left(G^{\prime} / A\right)) \cap G^{x x}$, it follows that the net $\left\{g\left(E_{F}\right): F \in \mathcal{F}\right\}$ must converge to the identity $I_{N}$. Nevertheless, $g\left(E_{F}\right)=\tilde{E}_{F}\left(\bar{\psi}_{B_{F}}(g)\right) \notin V$ for all $F \in \mathcal{F}$. This contradiction completes the proof. 
When $G=\sum_{i \in I} F_{i}$ is a direct sum, then it is not longer necessary to assume $G$ to be torsion. Indeed, a slight variation in the proof of Proposition 3.11 also gives the following result.

Corollary 3.12. Let $G=\sum_{i \in I} F_{i}$, where $F_{i}^{\prime}$ is finite for all $i \in I$. if we further assume that $\operatorname{mdus}\left(F_{i}\right) \leq N$ and $\exp \left(F_{i}{ }^{\prime}\right) \leq N$, for some $N \in \mathbb{N}$ and for all $i \in I$, then $G^{\prime}$ is Chu semi-reflexive in $G$.

Proof. Again, it is enough to take $\mathcal{B}=\left\{\sum_{i \in I \backslash J} F_{i}: J \subset I\right.$, and $J$ finite $\}$

Lemma 3.13. Let $G$ be a metrizable group such that $\epsilon_{G}(G)$ is dense in $G^{x x}$ and the groups $G$ and $\epsilon_{G}(G)$ have the same convergent sequences. Then $G^{x x}$ is Chu reflexive.

Proof. It follows from the hypothesis that the group $G$ is MAP. Indeed, take $g_{1} \neq g_{2}$ in $G$ such that $\epsilon_{G}\left(g_{1}\right)=\epsilon_{G}\left(g_{2}\right)$, then the sequence $\left\{x_{n}\right\}$ which alternates $g_{1}$ and $g_{2}$ is not convergent, but $\epsilon_{G}\left(x_{n}\right)$ is convergent which is impossible. Since the group $G$ is metrizable, by Proposition $2.1(1), G_{n}^{x}$ is hemicompact for all $n \in \mathbb{N}$, and thus the compact-open topology on $G^{x x}$ is metrizable. In particular, $\epsilon_{G}(G)$ is metrizable, and thus $G$ and $\epsilon_{G}(G)$ are topologically isomorphic, because they have the same convergent sequences. Therefore, $G^{x}=\left(\epsilon_{G}(G)\right)^{x}$. On the other hand, $\epsilon_{G}(G)$ is dense in $G^{x x}$, so by a recent result of Lukács (see [11]), $\left(\epsilon_{G}(G)\right)_{n}^{x}=G_{n}^{x x x}$. Hence, $G^{x}=\left(\epsilon_{G}(G)\right)^{x}=G^{x x x}$, and as a consequence $G^{x x}$ is Chu reflexive.

Corollary 3.14. Let $G$ be a semi-reflexive metrizable group such that $G$ and $G^{x x}$ have the same convergent sequences. Then $G$ is Chu.

Proof of Theorem 3.1. Propositions 3.3 and 3.11 yield the semi-reflexivity of $G$. In order to apply Corollary 3.14, we have to verify that $G$ and $G^{x x}$ have the same convergent sequences. Since the evaluation map is always continuous, it follows that every sequence converging in $G$ also converges in $G^{x x}$. So, let $\left\{\bar{g}_{m}\right\}$ be a sequence converging to the neutral element in $G^{x x}$. By hypothesis, there must be a co-countable normal subgroup $L$ of $G$ such that the canonical quotient $\pi_{L}: G \longrightarrow G / L$ is one-toone on the sequence $\left\{\bar{g}_{m}\right\}$. On the other hand, since every representation on $G / L$ can be extended canonically to $G$, we deduce that $\left\{\pi_{L}\left(\bar{g}_{m}\right)\right\}$ converges to the neutral element in $(G / L)^{x x}$. Now, the group $G / L$ is countable and, by the same logic as $G$, semi-reflexive . Hence, by Proposition 2.1 (6), $G / L$ is Chu. That is to say, $\left\{\pi_{L}\left(\bar{g}_{m}\right)\right\}$ converges in $G / L$ which is a discrete group. Hence, the injectivity of $\pi_{L}$ on the sequence $\left\{\bar{g}_{m}\right\}$ completes the proof.

Theorem 3.1 yields the following characterization of Chu reflexivity for direct sums of finite simple non-abelian groups.

Corollary 3.15. Let $G=\sum_{i \in I} F_{i}$, where $F_{i}$ is a finite simple non-abelian group for each $i \in I$. Then the group $G$ is Chu if and only if the $\operatorname{set}\left\{\exp \left(F_{i}\right): i \in I\right\}$ is bounded.

Proof. Assume that the set $\left\{\exp \left(F_{i}\right): i \in I\right\}$ is bounded. Let $X$ be a set of finite cardinality, $|X|$, greater than $\exp \left(F_{i}\right)$ for all $i \in I$. By Cayley's Theorem (see [18, 1.6.8]), every group $F_{i}$ is isomorphic to a subgroup of $S_{|X|}$, the symmetric group of 
degree $|X|$. Thus, the set $\left\{m d u s\left(F_{i}\right)\right\}$ is bounded. Finally, Corollary 3.2 yields that $G$ is Chu.

Conversely, suppose that $\left\{\exp \left(F_{i}\right): i \in I\right\}$ contains a countable subset, say $\left\{F_{i_{j}}\right.$ : $j \in \mathbb{N}\}$, such that $\exp \left(F_{i_{j}}\right) \geq j$ for all $j \in \mathbb{N}$. Since the groups $F_{i_{j}}$ are simple and non-abelian, it follows that if $\left[G_{n}^{x}, V\right]$ is an arbitrary canonical neighborhood of the identity in $G^{x x}$, for $V$ any neighborhood of the identity in $U(n)$, it holds that $\left[G_{n}^{x}, V\right]$ always contains a subgroup of the form $\sum_{j \geq m} F_{i_{j}}$, for some $m \in \mathbb{N}$. Indeed, applying Jordan's theorem (see [4]), given any $n \in \mathbb{N}$, there is a natural number $f(n)$ such that, for all $D \in G_{n}^{x}, D\left(F_{i_{j}}\right)=I_{n}$ if $j \geq f(n)$. This clearly implies that $\left[G_{n}^{x}, V\right]$ contains the subgroup $\sum_{j \geq f(n)} F_{i_{j}}$. This verifies that $G^{x x}$ is not discrete. Therefore, the group $G$ is not Chu.

Remark 3.16. Observe that, if $C>0$ is given and $\left\{F_{i}\right\}_{i \in I}$ is a family of finite simple groups, such that $\exp \left(F_{i}\right) \leq C$ for all $i \in I$, then there are finitely many $F_{i}$ 's up to isomorphism: this follows from the fact that every finite simple group is 2-generated, and from the positive solution to the restricted Burnside problem.

Following the terminology of Trigos-Arrieta, we say that a topological group $G$ respects a topological property $P$ if a subset $A$ of $G$ has $P$ as a subspace of $G$ if and only if $A$ has $P$ as a subspace of $b G$. In [16] (see also [3]) Remus and Trigos-Arrieta ask whether a Chu group $G$ always respects compactness. We notice that if $G$ is the weak sum of a countable family of finite simple non-abelian groups whose order is bounded then $G$ is Chu by Corollary 3.15. On the other hand, Corollary 3.10 implies that $b G=\prod_{i \in \mathbb{N}} F_{i}$ which is metrizable. This means that the discrete group $G$ contains non-trivial sequences that are convergent in $b G$. Thus, there are Chu groups that do not respect compactness.

The following examples follow from Theorem 3.1 Here on, the symbolism $H \rtimes K$ denotes the semi-direct product of the groups $H$ and $K$ where the latter is a subgroup of the automorphism group of the former.

Example 3.17. (Heyer 9]) Let $\mathbb{Z}_{3} \rtimes \mathbb{Z}_{2}=S_{3}$ the permutation group. Define $G_{i}=$ $\mathbb{Z}_{3} \rtimes \mathbb{Z}_{2}$ for all $i \in \mathbb{N}$ and take $G=\sum_{i \in \mathbb{N}} G_{i}$. Then $G$ is Chu reflexive.

That not every direct sum of finite groups is Chu was shown by Moran.

Example 3.18. (Moran [12]) Let $\left\{p_{i}\right\}$ be an infinite sequence of distinct prime numbers $\left(p_{i}>2\right)$, and let $F_{i}$ be the projective special linear group of dimension two over the Galois field $G F\left(p_{i}\right)$ of order $p_{i}$. It holds that $G=\sum_{i \in \mathbb{N}} F_{i}$ is not Chu.

The methods of Theorem 3.1 can also be applied to some non discrete groups with slight modifications. Next follows an example of this fact. Let $\left\{G_{i}: i \in I\right\}$ be a non void family of locally compact groups and set $G=\prod_{i \in I} G_{i}$. Let $H_{i}$ be an open subgroup of $G_{i}$ for all $i \in I$. The local direct product of the $G_{i}$ 's relative to the open subgroups $H_{i}$ is defined as the subgroup $G_{0}$ of $G$ consisting of all $\left(x_{i}\right)$ for which $x_{i} \in H_{i}$ for all but a finite number of indices $i$, and equipped with the following topology: Let $J$ be a finite subset of $I$, and let $U_{i}$ be a neighborhood of $1_{i}$ in the subgroup $H_{i}$ for each $i \in J$; let the sets $\prod_{i \in J} U_{i} \times \prod_{i \in I \backslash J} H_{i}$ be taken as an open basis at the neutral element $\left(1_{i}\right)$ in $G_{0}$. It follows that $G_{0}$ is a topological group containing the subgroup 
$H=\prod_{i \in I} H_{i}$ as an open subgroup. It is readily seen that $G_{0}$ is locally compact if the subgroup $H_{i}$ is compact for all $i \in I$. We have the following consequence of Corollary 3.2 .

Corollary 3.19. Let $G_{0}$ be the local direct product of the family $\left\{G_{i}, H_{i}\right\}$ where $H_{i}$ is a compact normal subgroup of $G_{i}$ such that $G_{i}^{\prime} / H_{i}$ is finite for all $i \in I$. Suppose further that $\operatorname{mdus}\left(G_{i} / H_{i}\right) \leq N$ and $\exp \left(G_{i}{ }^{\prime} / H_{i}\right) \leq N$, for some $N \in \mathbb{N}$ and for all $i \in I$. Then $G_{0}$ is Chu semi-reflexive. Moreover, if $I$ is countable, the group $G_{0}$ is Chu reflexive.

Proof. Observe that $H=\prod_{i \in I} H_{i}$ is a compact-open subgroup of $G_{0}$. Hence, $G_{0} / H$ is discrete and thus topologically isomorphic to $\sum_{i \in I} G_{i} / H_{i}$. The collection $\left\{G_{i} / H_{i}\right.$ : $i \in I\}$ satisfies the conditions of Theorem 3.1, so its (weak-)sum is Chu semi-reflexive. Therefore $G_{0} / H$ is semi-reflexive. Since $H$ is compact, it is trivially Chu semi-reflexive in G. Now, it suffices to apply Proposition 3.3 to obtain that $\mathrm{G}$ is semi-reflexive. Finally, in case $I$ is countable, the local direct product $\left\{G_{i}, H_{i}\right\}$ is second countable and locally compact. Hence, the reflexivity follows immediately from item (6) of Proposition 2.1.

Corollary 3.19 does not hold if we remove the constraint of being $H_{i}$ a normal subgroup of $G_{i}$.

Example 3.20. Take the permutation group $S_{3}=\mathbb{Z}_{3} \rtimes \mathbb{Z}_{2}$, let $G_{i}=\mathbb{Z}_{3} \rtimes \mathbb{Z}_{2}$ and $H_{i}=\mathbb{Z}_{2}$ for all $i \in \mathbb{N}$. If we consider the local direct product $G_{0}$ of the family $\left\{G_{i}, H_{i}\right\}_{i \in \mathbb{N}}$, then $G_{0}$ is not Chu reflexive.

Proof. Indeed, using that the smallest normal subgroup that contains $H_{i}$ is $G_{i}$, it can be proved that the spaces $\left(G_{0}\right)_{n}^{x} / \sim$ are discrete for all $n \in \mathbb{N}$. Then Proposition 2.2 yields that $G_{0}^{x x}$ is topologically isomorphic to $\prod_{i \in \mathbb{N}} G_{i}$.

\section{TAKAHASHI GROUPS}

For each locally compact group $G$, Takahashi has constructed a locally compact group $G_{T}$ called Takahashi quasi dual such that $G_{T}$ is maximally almost periodic, and $G_{T}^{\prime}$ is compact. The category of locally compact groups with these two properties is denoted by TAK. If $n>1$ and $D \in \operatorname{Hom}_{c}(G, U(n))$ then the sets $t_{n}(D ; U)=$ $\{D \otimes \chi: \chi \in U\}, U$ any neighborhood of the identity in the group $G_{1}^{x}$, form a fundamental system of neighbourhoods of $D$ for a topology in $H_{c} m_{c}(G, U(n))$. We denote by $G_{n}^{t}$ the set $H_{o m}(G, U(n))$ equipped with this topology and the symbol $G^{t}$ denotes the topological sum of the spaces $G_{n}^{t}$, for $n \in \mathbb{N}$. A unitary mapping on $G^{t}$ is a continuous mapping $p: G^{t} \longrightarrow \mathcal{U}$ conserving the main operations between unitary representations: direct sums, tensor products, unitary equivalence and sending the the elements of $G_{n}^{t}$ into $U(n)$ for all $n \in \mathbb{N}$ (see [13] for details). The set of all unitary mappings on $G^{t}$ equipped with the compact-open topology is a topological group, with pointwise multiplication as the composition law, which called the Takahashi quasidual group of $G$ and is denoted by $G_{T}$. It is easily verified that $G^{x x} \subset G_{T} \subset b G$. On the other hand, the evaluation map defines a group homomorphism $\epsilon_{G}^{T}: G \longrightarrow G_{T}$, which is a monomorphism if and only if $G$ is MAP. The group $G$ satisfies Takahashi duality when $\epsilon_{G}^{T}$ is an isomorphism of topological groups. The Takahashi duality theorem establishes that $G$ satisfies this duality if $G \in$ TAK . It is known that LCA 
groups and compact groups belong to TAK. (cf. [20]). On the other hand, given any group $G$, we have that $\epsilon_{G}(G)$ is dense in $G_{T}$ and for each $H \in$ TAK and each homomorphism $f: G \longrightarrow H$ there exists exactly one homomorphism $f^{t}: G_{T}: \longrightarrow H$ with $f=f^{t} \circ \epsilon_{G}$. A detailed discussion and extension of this theory has been given by Poguntke in [13, from where we have taken these lines.

In this section, we are interested in finding out when the Chu quasi-dual coincides with the Takahashi quasi-dual for MAP groups. Firstly, we give some examples that illustrate the different situations arising in the theory.

Example 4.1. (Moran [12]) Let $\left\{p_{i}\right\}$ be an infinite sequence of distinct prime numbers $\left(p_{i}>2\right)$, and let $F_{i}$ be the projective special linear group of dimension two over the Galois field $G F\left(p_{i}\right)$ of order $p_{i}$. If $G=\sum_{i \in \mathbb{N}} F_{i}$, we have $G^{x x}=G_{T}=b G$.

Example 4.2. (Heyer [9]) Let $\mathbb{Z}_{3} \rtimes \mathbb{Z}_{2}=S_{3}$ the permutation group. Define $G_{i}=\mathbb{Z}_{3} \rtimes$ $\mathbb{Z}_{2}$ for all $i \in \mathbb{N}$ and take $G=\sum_{i \in \mathbb{N}} G_{i}$. Then $G=G^{x x}$ and $G_{T}=\prod_{i \in \mathbb{N}} \mathbb{Z}_{3} \rtimes \sum_{i \in \mathbb{N}} \mathbb{Z}_{2}$.

Proof. It was proved by Heyer 9] that $G=G^{x x}$. On the other hand, by Corollary 3.10 $c l_{b G} G^{\prime}=\prod_{i \in \mathbb{N}} \mathbb{Z}_{3}$. It suffices now to use [13, Prop. 17] to complete the proof.

Example 4.3. Let $p$ a prime number greater than 2, and let $F_{i}$ be the projective special linear group of dimension two over the Galois field $G F(p)$ of order $p$. If $G=\sum_{i \in \mathbb{N}} F_{i}$, we have $G^{x x}=G$ and $G_{T}=b G$.

Proof. Applying Corollary [3.2, we know that $G^{x x}=G$ and, by Corollary [3.10, we have $b G=c l_{b G} G^{\prime}=\prod_{i \in \mathbb{N}} F_{i}$. Again, it suffices to apply [13, Prop. 17] and the proof is done.

If $G$ is a locally compact group and $G^{\prime}$ denotes its commutator subgroup, we define the following equivalence relation $\mathcal{R}$ on $\widehat{G}_{n}$. Let $\sigma, \tau \in \widehat{G}_{n}$, then $\sigma \mathcal{R} \tau$ if and only if there are two representations $D \in \sigma, E \in \tau$ and an unitary matrix $U \in U(n)$ such that $D(x)=U E(x) U^{-1}$ for all $x \in G^{\prime}$. Since each equivalence class defined by $\sim$ is clearly contained in some equivalence class defined by $\mathcal{R}$, it is easily verified that $\mathcal{R}$ defines a closed equivalence relation on $\widehat{G}_{n}$. Hence, there is a canonical quotient mapping $\bar{\pi}: \widehat{G}_{n} \longrightarrow \widehat{G}_{n} / \mathcal{R}$, which is continuous. On the other hand, we note that, using essentially the same definition, the equivalence relation $\mathcal{R}$ can also be defined on the spaces $\operatorname{Irr}_{n}(G)$ and we obtain a similar quotient mapping $\tilde{\pi}: \operatorname{Irr}_{n}(G) \longrightarrow \operatorname{Irr}_{n}(G) / \mathcal{R}$. If we define $p: \frac{\operatorname{Irr}_{n}(G)}{\mathcal{R}} \longrightarrow \frac{\widehat{G}_{n}}{\mathcal{R}}$ so that the following diagram

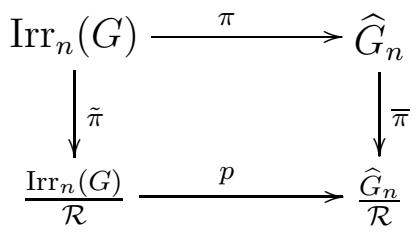

commutes, then it is easy to verify that the map $p$ is actually a homeomorphism. Using this observation, we can state now the main result of this section follows.

Theorem 4.4. Let $G$ be a locally compact group. We have that $G^{x x}$ is topologically isomorphic to $G_{T}$ if and only if $\widehat{G}_{n} / \mathcal{R}$ is discrete for all $n \in \mathbb{N}$.

Firstly, we need some preliminary results. 
Lemma 4.5. Let $G$ be a Takahashi group. Then $\widehat{G}_{n} / \mathcal{R}$ is discrete for all $n \in \mathbb{N}$

Proof. According to the definition of $\mathcal{R}$, we have a commutative diagram

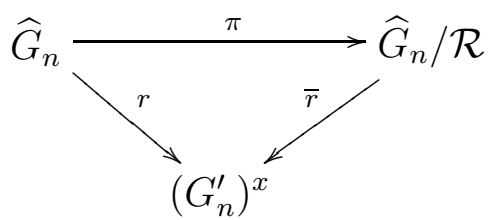

where, for each $\sigma \in \widehat{G}_{n}$ and $D \in \sigma$, the class $r(\sigma)$ is defined by the representation $D_{\mid G^{\prime}} \in \operatorname{Hom}\left(G^{\prime}, U(n)\right)$. On the other hand, the map $\bar{r}$ is defined by the equality $\bar{r} \circ \pi=r$. It is readily seen that $\bar{r}$ is continuous and injective. Now, since $G^{\prime}$ is a compact group, we have that $\left(G_{n}^{\prime}\right)^{x}$ is discrete. Hence, its inverse image $\widehat{G}_{n} / \mathcal{R}$ is also discrete.

Lemma 4.6. Let $G$ be a maximally almost periodic, locally compact group such that $\left(G^{x x}\right)^{\prime}$ is a compact subgroup of $G^{x x}$. Then $G / G^{\prime}$ is topologically isomorphic to $G^{x x} /\left(G^{x x}\right)^{\prime}$.

Proof. Consider the following diagram, where $\epsilon_{G}$ is the natural injection of $G$ into $G^{x x}$ and the other mappings are defined in canonical way.

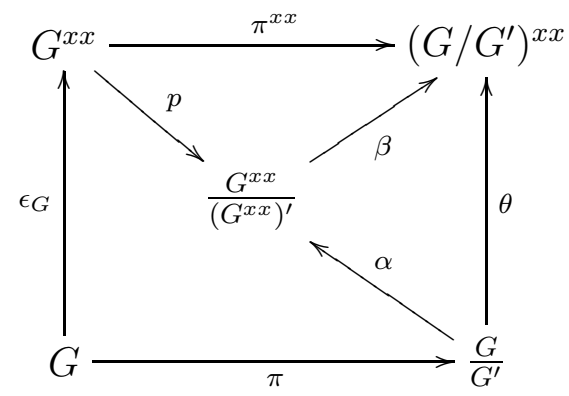

Let $H$ be the kernel of $\beta$ and let $\gamma: G^{x x} \longrightarrow b G$ be the natural inclusion (here, we view $G^{x x}$ as a subgroup of $\left.b G\right)$. We wish to show $\gamma(H) \subset b(G)^{\prime}$. First, we note that, since $(b G)^{\prime}$ is the intersection of kernels of all unitary characters of $b G$ we have $\overline{b\left(G^{\prime}\right)}=(b G)^{\prime}$ for every discrete group $G$. Now, since $\left(G^{x x}\right)^{\prime} \supseteq \epsilon_{G}\left(G^{\prime}\right)$, we have $\overline{\left(G^{x x}\right)^{\prime}} \supseteq \overline{\epsilon_{G}\left(G^{\prime}\right)}$. Hence, $\overline{\gamma\left(\overline{\left(G^{x x}\right)^{\prime}}\right)} \supseteq \overline{\gamma\left(\epsilon_{G}\left(G^{\prime}\right)\right)}=\overline{b\left(G^{\prime}\right)}$. As $\left(G^{x x}\right)^{\prime}$ is compact, we obtain $\gamma\left(\left(G^{x x}\right)^{\prime}\right)=(b G)^{\prime}$. Now, for each $h \in H, \beta(h)$ is the identity of $\left(\frac{G}{G^{\prime}}\right)^{x x}$; i.e., for each finite-dimensional unitary representation $D: \frac{G}{G^{\prime}} \longrightarrow U(d(D))$, we have $\beta(h)(D)=I_{d(D)}$. Thus, if $\pi: G \longrightarrow \frac{G}{G^{\prime}}$ is the canonical quotient mapping, it holds $h(D \circ \pi)=I_{d(D)}$. Suppose $h \in H$ and $\gamma(h) \notin(b G)^{\prime}$; then there is a finite-dimensional unitary representation $E$ of $b G /(b G)^{\prime}$ such that $E\left(\gamma(h)(b G)^{\prime}\right) \neq I_{d(E)}$. Hence, if $b_{G^{\prime}}: \frac{G}{G^{\prime}} \longrightarrow \frac{b G}{(b G)^{\prime}}$ denotes the inclusion canonically associated to $b: G \longrightarrow b G$, we have $h\left(E \circ b_{G^{\prime}} \circ \pi\right) \neq I_{d(E)}$. This is a contradiction; therefore, we have $\gamma(H) \subset$ $(b G)^{\prime}=\gamma\left(\left(G^{x x}\right)^{\prime}\right)$. This implies that the kernel of $\beta$ is trivial. On the other hand, since $G / G^{\prime}$ is Abelian, the map $\theta$ is a surjective topological isomorphism. So, by the commutativity of the diagram, the map $\alpha: \frac{G}{A} \longrightarrow \frac{G^{x x}}{\left(G^{x x}\right)^{\prime}}$ is a topological isomorphism as well, which completes the proof. 
Proof of Theorem 4.4. Let us suppose that $G_{T}=G^{x x}$ and consider the diagram

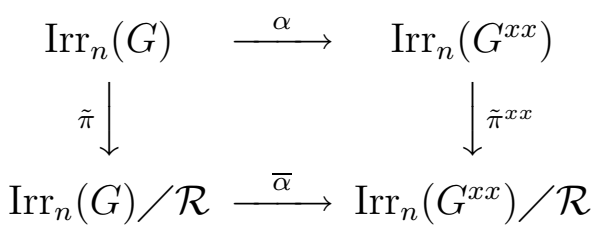

where $\alpha: \operatorname{Irr}_{n}(G) \longrightarrow \operatorname{Irr}_{n}\left(G^{x x}\right)$ is the canonical evaluation map defined by $\alpha(D)(x)=$ $x(D)$ for all $D \in \operatorname{Irr}_{n}(G)$ and $x \in G^{x x}$. We know that $\alpha$ is continuous and injective (see Proposition 2.2). On the other hand, the map $\bar{\alpha}$ is defined so that the diagram commutes. Since $G^{\prime}$ is dense in $G_{T}^{\prime}=\left(G^{x x}\right)^{\prime}$, it follows that $\bar{\alpha}$ is also well defined, continuous and injective. Then we set the diagram

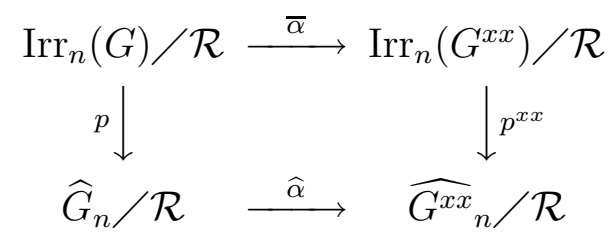

where, for any $\sigma \in \widehat{G}_{n} / \mathcal{R}$, the element $\widehat{\alpha}(\sigma)$ is defined as the class of $\bar{\alpha}(D)$ with $D \in \sigma$, which makes commutative the diagram. Again, it is readily verified that $\widehat{\alpha}$ is well defined, continuous and injective. Since $\left(G^{x x}\right)^{\prime}$ is compact, we get by Lemma 4.5 that $\widehat{G^{x x}}{ }_{n} / \mathcal{R}$ is discrete, which implies that $\widehat{G}_{n} / \mathcal{R}$ is discrete.

Conversely, let us suppose that $\widehat{G}_{n} / \mathcal{R}$ is discrete for all $n \in \mathbb{N}$. Since $G$ is dense in $G^{x x}$ with respect to the Bohr topology (that is to say, when they are identified to subgroups of $b G$ ), it follows that for every $D$ and $E$ in $H_{o m}(G, U(n))$ with $D \mathcal{R} E$, there is a unitary matrix $U$ such that $D(x)=U E(x) U^{-1}$ for all $x \in\left(G^{x x}\right)^{\prime}$. Then, repeating an argument similar to the one used in [6. Th. 4.4], we obtain that $\left(G^{x x}\right)^{\prime}$ is totally bounded as a (topological) subgroup of $G^{x x}$. The latter group is a complete uniform space, which yields $\left(G^{x x}\right)^{\prime}$ is compact and topologically isomorphic to $\mathrm{cl}_{b G} G^{\prime}$. Hence, we have verified that $G^{x x}$ is a Takahashi group. On the other hand, Lemma 4.6] asserts that $G^{x x} /\left(G^{x x}\right)^{\prime}$ is topologically isomorphic to $G / G^{\prime}$. From this fact, it follows that the canonical mapping $\epsilon_{G}: G \longrightarrow G^{x x}$ is a dense continuous injection. Now, consider the diagram

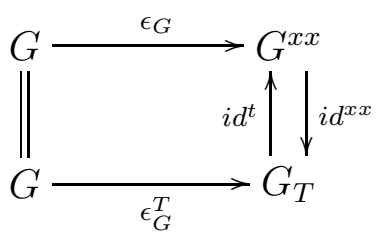

Since $\epsilon_{G}$ and $\epsilon_{G}^{T}$ are dense continuous injections into Takahashi groups, we have that $G^{x x}$ and $G_{T}$ are canonically isomorphic, which completes the proof.

Corollary 4.7. Let $G$ be a simple $M A P$ discrete group (which implies $G^{\prime}=G$ ). Then the following conditions are equivalent:

(i) $G^{x x}=G_{T}$;

(ii) $\widehat{G}_{n}$ is discrete for all $n \in \mathbb{N}$;

(iii) $G^{x x}=b G$. 
Proof. (i) $\Rightarrow$ (ii) Since $G^{\prime}=G$, the relation $\mathcal{R}$ coincides with $\sim$. Therefore, it is enough to apply Theorem 4.4

(ii) $\Rightarrow$ (iii) This is [6, Theorem 4.4].

(iii) $\Rightarrow$ (i) It follows from the fact that $G^{x x}$ can be injected canonically into $G_{T}$ and the latter can be injected into $b G$.

Corollary 4.8. Let $G$ be a discrete MAP group that is nilpotent of length two, and such that for each positive integer $n$ there are only finitely many co-finite normal subgroups $H$ of $G^{\prime}$ whose index is less or equal than $n$. Then $G^{x x} \cong G_{T}$.

Proof. We first verify that, for each $n \in \mathbb{N}$, there is a finite subgroup $F$ of $U(n)$ such that, given any $D \in \operatorname{Irr}_{n}(G)$, we have $D\left(G^{\prime}\right) \subset F$. Indeed, by Lie, Kolchin, Mal'cev's theorem (see [18, 15.1.1]), there is an Abelian subgroup $A$ of $D(G)$ with finite index, say $m$. That is, $D\left(g^{m}\right) \in A$ for all $g \in G$. Now, for $x$ and $y$ arbitrarily taken in $G$, since $G^{\prime}$ is central in $G$, we have $(D[x, y])^{m^{2}}=[D(x), D(y)]^{m^{2}}=\left[D(x)^{m}, D(y)^{m}\right]=1$. This yields $\exp D\left(G^{\prime}\right) \leq m^{2}$ for all $D \in \operatorname{Irr}_{n}(G)$. Hence, it suffices to take $F$ to be the subgroup consisting of all $\mathrm{m}^{2}$-roots of the identity in $U(n)$.

Thus, fixed any positive integer $n$, we may find $m(n) \in \mathbb{N}$ with $\left[G^{\prime}:(\operatorname{ker} D) \cap G^{\prime}\right] \leq$ $m(n)$ for all $D \in \operatorname{Irr}_{n}(G)$. By hypothesis, the are only finitely many co-finite normal subgroups of $G^{\prime}$ whose index is less or equal than $m(n)$. Therefore, it follows that $\widehat{G}_{n} / \mathcal{R}$ is finite for all $n \in \mathbb{N}$ and the proof is complete by Theorem 4.4.

As a consequence, we obtain the following result due to Poguntke [14, 15].

Corollary 4.9. (Poguntke, 1976) The Heisenberg integral group H, satisfies that $H^{x x} \cong H_{T}$.

Theorem 4.10. Let $G$ be a discrete $M A P$ group that is an $F C$ group and, for each positive integer $n$, there are only finitely many co-finite normal subgroups $H$ of $G^{\prime}$ such that $G^{\prime} / H$ accepts faithful representations into $U(n)$. Then $G^{x x} \cong G_{T}$.

Proof. Given $n \in \mathbb{N}$ and $D$ arbitrarily chosen in $G_{n}^{x}$, we know by Corollary [3.7 that $G^{\prime} \cap \operatorname{ker} D$ is a co-finite normal subgroup of $G^{\prime}$. Since, there are a finite number of such subgroups, we can take $L$ to be the intersection of all them. Thus, $\left(G_{n}^{x}\right)_{\mid G^{\prime}}$ can be injected, as a set, into $\left(G^{\prime} / L\right)_{n}^{x}$. Now, the set $\widehat{\left(G^{\prime} / L\right)_{n}}$ is trivially finite. This implies that $\widehat{G}_{n} / \mathcal{R}$ is finite for all $n \in \mathbb{N}$. The proof is completed by applying Theorem 4.4 .

Corollary 4.11. Let $G=\sum_{n \in \mathbb{N}} F_{n}$, where each $F_{n}{ }^{\prime}$ is simple and $\lim _{n \rightarrow \infty} \exp \left(F_{n}{ }^{\prime}\right)=$ $\infty$. Then $G^{x x} \cong G_{T}$.

\section{Some General Remarks}

The results stated in the sections above establish a classification of countable discrete MAP groups according to their unitary representation spaces. Let $G$ be a countable discrete MAP group. From the space of finite dimensional unitary representations, we have the following cases:

(i) There is an integer $n$ and a representation $D \in G_{n}^{x}$ which is faithful. In this case $G$ is a subgroup of $U(n)$, equipped with the discrete topology. 
(ii) There is an integer $n$ such that $G_{n}^{x}$ separates the points of $G$ but no representation on $G$ is faithful. For each $D \in G_{n}^{x}$, let $N(D)$ be the kernel of $D$. We may form the group $\prod_{D \in G_{n}^{x}} G / N(D)$. Since $G_{n}^{x}$ separates points, the group $G$ may be viewed as a subgroup of $\prod_{D \in G_{n}^{x}} G / N(D)$. Examples of these groups are weak direct sums of finite groups with bounded exponent equipped with the discrete topology.

(iii) There is no integer $n$ such that $G_{n}^{x}$ separate the points of $G$. For example $\sum G F\left(p_{n}\right)$ for various primes $p_{n}$ or the Heisenberg integral group. In this case we have that $G$ is never a Chu group. Indeed, given any integer $n$, there are infinitely many points which belong to $\left[G_{n}^{x}, V\right]$ for any $V$ neighborhood of the identity in $U(n)$. As a consequence, the topology on $G$ inherited from $G^{x x}$ is not discrete. Hence, the group is not $\mathrm{Chu}$.

For next result, we need some preparation. Let $G$ and $H$ be topological groups and let $f$ be a continuous homomorphism from $G$ to $H$. Then $f$ induces canonically the maps $f_{n}^{x}: H_{n}^{x} \longrightarrow G_{n}^{x}, f^{x}: H^{x} \longrightarrow G^{x}$, and $f^{x x}: G^{x x} \longrightarrow H^{x x}$. Let $G$ be locally compact and let $\left\{F_{i}\right\}$ be a collection of compact normal subgroups such that $F_{i} \supseteq F_{j}$ if $i \geq j, \cap F_{i}=\left\{1_{G}\right\}$, and $G=\underset{\operatorname{Lim}}{\longleftarrow} G_{i}$ (with $G_{i}=G / F_{i}$ ). Suppose that $H$ has a neighborhood $V$ of the identity which contains no small subgroups of $H$ other than $\left\{1_{H}\right\}$. Then, if $f: G \longrightarrow H$ is any continuous homomorphism, there is and index $i_{0}$ such that for $i \geq i_{0}$ there exists a continuous homomorphism $f_{i}: G_{i} \longrightarrow H$ such that $f=f_{i} \circ \phi_{i}$, where $\phi_{i}$ is the canonical homomorphism of $G$ onto $G_{i}$ (cf. [1, Chap. 3, p. 294]).

We shall make use of the following lemma whose proof is left to the reader.

Lemma 5.1. Let $G$ and $H$ be locally compact groups and let $f: G \longrightarrow H$ a continuous group homomorphism. Then the diagram

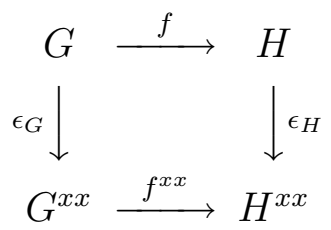

commutes.

Proposition 5.2. Let $G=\underline{\operatorname{Lim}} G_{i}$ be a locally compact group that is the projective limit of Chu semi-reflexive groups. Suppose further that each canonical projection $\phi_{i}: G \longrightarrow G_{i}$ is surjective with compact kernel. Then $G$ is Chu semi-reflexive. If, in addition, the group $G$ is second countable, then $G$ is a Chu group.

Proof. Since each $G_{i}$ is Chu and $G$ is embedded into their product, it follows that $G$ is MAP. We now show that the evaluation map $\epsilon_{G}$ is surjective. Let $p$ be an element in $G^{x x}$. For each $i, \phi_{i}^{x x}(p)=\epsilon_{G_{i}}\left(x_{i}\right)$, with $x_{i} \in G_{i}$. Let $x$ be the element in the product $\prod_{i \in I} G_{i}$ defined by $\left(x_{i}\right)_{i \in I}$. Using Lemma 5.1, it is readily seen that $x$ belongs to $G$. Now, if $D$ is an arbitrary element of $G^{x}$, by the remark above, there is an index $i_{0}$ such that for each $i \geq i_{0}$, there exists $D_{i} \in G_{i}^{x}$ such that $D=D_{i} \circ \phi_{i}$. Thus, $p(D)=p\left(D_{i} \circ \phi_{i}\right)=p\left(\phi_{i}^{x}\left(D_{i}\right)\right)=\phi_{i}^{x x}(p)\left(D_{i}\right)=\epsilon_{G_{i}}\left(x_{i}\right)\left(D_{i}\right)$. By the way in which $x$ was defined, this yields $p(D)=\epsilon_{G}(x)(D)$. Therefore $p=\epsilon_{G}(x)$ and $\epsilon_{G}$ is surjective. 
Finally, in case that $G$ is second countable, it suffices to apply Proposition 2.1$]$ to conclude that $G$ is a $\mathrm{Chu}$ group.

Next example shows that the Chu quasi-dual group $G^{x x}$ need not be locally compact even for a countable discrete group $G$.

Example 5.3. Let $\left\{p_{n}\right\}$ be an infinite sequence of distinct prime numbers $\left(p_{n}>2\right)$, and let $G_{n}=P S L\left(2, p_{n}\right)$ be the projective special group of dimension two over the finite filed of order $p_{n}$. For each $n$, let $G_{n, m}$ be a copy of $G_{n}$, for $m=1,2, \ldots$. Let $G=\sum_{n=1}^{\infty}\left(\sum_{m=1}^{\infty} G_{n, m}\right)$ with the discrete topology. The group $G^{x x}$ is not locally compact.

Proof. Indeed, let $V$ be a small neighborhood of the identity in $U(n)$. Let $\left[G_{n}^{x}, V\right]$ be the neighborhood in $G^{x x}$ defined by $\left[G_{n}^{x}, V\right]=\left\{p \in G^{x x}: p\left(G_{n}^{x}\right) \subset V\right\}$. Observe that $\epsilon_{G}\left(\sum_{m=1}^{\infty} G_{l, m}\right) \subset\left[G_{n}^{x}, V\right]$ if $l$ is sufficiently large. On the other hand, applying Corollary [3.2. we know that $\sum_{m=1}^{\infty} G_{l, m}$ is Chu. Using this and, since the group $G$ projects canonically onto $\sum_{m=1}^{\infty} G_{l, m}$, it follows that the latter discrete group is topologically isomorphic to $\epsilon_{G}\left(\sum_{m=1}^{\infty} G_{l, m}\right)$. Hence, $G^{x x}$ can not be locally compact (since no compact set can contain an infinite discrete subgroup).

Acknowledgement: We would like to thank Gábor Lukács for several useful remarks. We also want to thank the referee for his/her constructive report. They have helped us to improve parts of this paper.

\section{REFERENCES}

[1] N. Bourbaki, Topologie générale, Chap. 1-4. Hermann. Paris. 1971.

[2] H. Chu, Compactification and duality of topological groups, Trans. Amer. Math. Soc. 123 (1966), 310-324.

[3] W. W. Comfort, Salvador Hernández, Dieter Remus, and F. Javier Trigos-Arrieta, Some open problems on topological groups, Proc. Workshop on topological groups and Lie groups (Madrid, 1999), Heldermann, Berlin, 2001, 57-76.

[4] C. W. Curtis and I. Reiner, Representation Theory of Finite Groups and Associative Algebras, Interscience Publishers. 1962. Reprinted in 1988.

[5] M. Enock and J. M. Schwartz, Kac Algebras and Duality of Locally Compact Groups, SpringerVerlag, Berlin - Heidelberg - New York. 1992.

[6] J. Galindo and S. Hernández, Interpolation sets and the Bohr topology of locally compact groups, Advances in Math., 188 (2004), 51-68.

[7] M. Goto, Note on a topology of a dual space, Proc. Amer. Math. Soc. 12 (1961), 41-46.

[8] H. Heyer, Dualität lokalkompakter Gruppen, Lecture Notes in Mathematics 150. SpringerVerlag. Berlin-Heidelberg-New York. 1970.

[9] H. Heyer, Groups with Chu duality. In: Probability and Information Theory, Lecture Notes in Mathematics (Springer-Verlag) 296 (1973), 181-215.

[10] G. Hochschild and D. G. Mostow Representations and representative functions of Lie groups, Ann. of Math. 66 (2) (1957), 495-542.

[11] G. Lukács, On homomorphism spaces of metrizable groups, J. Pure and Appl. Algebra 182 (2-3) (2003), 263-267.

[12] W. Moran, On almost periodic compactifications of locally compact groups, J. London Math. Soc. 3 (2) (1971), 507-512.

[13] D. Poguntke, A universal property of the Takahashi quasi-dual, Can. J: Math., XXIV (3) (1972), 530-536.

[14] D. Poguntke, Zwei Klassen lokalkompakter maximal fastperiodischer Gruppen, Monatsh. Math. 81 (1976), 15-40. 
[15] D. Poguntke, Chu-Dualität und zwei Klassen maximal fastperiodischer Gruppen, Monatsh. Math. 82 (1976), 31-50.

[16] D. Remus and F. J. Trigos-Arrieta, The Bohr topology of Moore groups, Top. Appl. 97 (1999), 85-98.

[17] L. Riggins, On infinite groups and unitary duality, Ph.D. thesis, Case Western Reserve University, Cleveland, Ohio, 1998.

[18] D.J.S. Robinson, A Course in the Theory of Groups, Springer-Verlag, New York (1982).

[19] D.W. Roeder, A characterization of unitary duality, Trans. Amer. Math. Soc., 148 (1970), 124-135.

[20] S. Takahashi, A duality theorem for representable locally compact groups with compact commutator subgroup, Tôhoku Math. J., 4 (1952), 115-121.

[21] T.S. Wu and L. Riggins, Maximally Almost Periodic Groups and a Theorem of Glicksberg, Annals of N. Y. Acad. of Scienc., 806 (1996), 454-464. 
Departamento de Matemáticas,

ÁreA CiEnTÍFICO-TÉCNICA,

UNIVERSIDAD JAUME I, 8029 - AP CASTEllón, SPAIN.

E-mail address: hernande@mat.uji.es

Department of MATHEMATics, Case Western Reserve University, Cleveland, Ohio 44106 , U.S.A.

E-mail address: txw3@po.cwru.edu 